\title{
ON THE RESIDUAL FINITENESS OF PERMUTATIONAL PRODUCTS OF GROUPS
}

\author{
R. B. J. T. ALLENBY
}

(Received 20 January 1969)

Communicated by G. E. Wall

\section{Introduction}

In a recent paper Gregorac [1] has posed the following question: if $P$ is a permutational product of the amalgam $\mathscr{A}=A \cup B \mid H$ and if $P$ is residually finite are all the permutational products of $\mathscr{A}$ residually finite?

The present note gives an example answering the question in the negative.

The notation will be that introduced by B. H. Neumann in [3]. In particular for elements $a \in A, b \in B, \rho(a), \rho(b)$ will denote the corresponding permutations in the permutational product under consideration. We use \{\} to denote groups, $\langle>$ for sets, ( ) for the permuted symbols of the permutational products and also for permutations themselves.

I should like to thank the referee for his comments.

\section{Preliminaries}

In our example below we shall need the following information which is easily deduced from $\S 4$ of B. H. Neumann [3].

Let

$$
\begin{aligned}
& X=\left\{x, k ; x^{3}=k^{2}=(x k)^{2}=1\right\}, \\
& Y=\left\{y, k ; y^{3}=k^{2}=(y k)^{2}=1\right\}, \\
& K=\left\{k ; k^{2}=1\right\}
\end{aligned}
$$

and let $\mathscr{X}$ be the amalgam $X \cup Y \mid K$. Take $X_{1}=\left\langle k, x, x^{2}\right\rangle$ as a set of left coset representatives of $X$ modulo $K$ and $Y_{1}, Y_{2}$ as the sets $\left\langle 1, y, y^{2}\right\rangle$ and $\left\langle k, y, y^{2}\right\rangle$ respectively of left coset representatives of $Y$ modulo $K$. If $P_{1}$ is the permutational product of $\mathscr{X}$ using $X_{1}$ and $Y_{1}$ then in $P_{1} \rho(x)$ and $\rho(y)$ generate a subgroup $S_{1}$ of permutations which is soluble of length 2 . If $P_{2}$ is the permutational product of $\mathscr{X}$ using $X_{1}$ and $Y_{2}$ then $P_{2} \cong S_{2} \times Z_{2}$ where $S_{2}=\{\rho(x), \rho(y)\} \cong A_{9}$, the alternating group on nine symbols, and $Z_{2}$ is a cycle of order 2 . 


\section{The example}

We construct $\mathscr{A}$ as follows. Take

and

$$
\begin{aligned}
& A=\left\{a, h ; a^{3}=h^{2}=(a h)^{2}=1\right\}, \\
& B=\left\{h, b_{1}, b_{2}, \cdots ; h^{2}=b_{\iota}^{3}=\left(h b_{\iota}\right)^{2}=\left[b_{\iota}, b_{j}\right]=1\right\} \\
& H=\left\{h ; h^{2}=1\right\} .
\end{aligned}
$$

To construct a permutational product $Q_{1}$ of $\mathscr{A}$ which is residually finite choose, as (left) coset representatives of $A$ modulo $H$, the elements $1, a, a^{2}$ and, as (left) coset representatives of $B$ modulo $H$, the elements of $C=\left\{b_{1}, b_{2}, \cdots\right\}<B$. It is easy to check that in $Q_{1}$ the equation $\left[\rho(a), \rho\left(b_{\imath}\right)\right]=1$ holds for all $b_{\imath}$. Hence $W_{1}=\left\{\rho(a), \rho\left(b_{1}\right), \rho\left(b_{2}\right), \cdots\right\}$ is the direct product of a countable infinity of 3-cycles and is consequently residually finite (see Lemma 1.1 [2]). Further $W_{1}$ has index 2 in $Q_{1}$ and so, as an extension of a residually finite group by a finite group, $Q_{1}$ is residually finite (Lemma 1.5 [2]).

To obtain a permutational product $Q_{2}$ of $\mathscr{A}$ which is not residually finite we choose, as coset representatives of $A$ and $B$ modulo $H$, the same systems of representatives as above except that we choose the representative of $H$ in each system to be ' $h$ ' rather than ' 1 '. To prove $Q_{2}$ is not residually finite we show that $Q_{2}$ contains a subgroup isomorphic to the alternating group on a countable infinity of symbols. Clearly no residually finite group can contain an infinite simple group.

Let $C$ be as above. First we select an arbitrary generator $b_{\iota}$ in $C$ and split the set of coset representatives of $B$ modulo $H$ into disjoint subsets $\left\langle h, b_{\iota}, b_{\iota}^{2}\right\rangle$, $\left\langle t, t b_{i}, t b_{\imath}^{2}\right\rangle$ where $t$ ranges over all non-identity elements of $C_{\iota}=\left\{b_{1}, b_{2}, \cdots\right.$, $\left.b_{t-1}, b_{\imath+1}, \cdots\right\}<C$. We now fix on such an element $t$ and consider the effect of the elements $\rho(a), \rho\left(b_{l}\right)$ firstly on the set of triplets $(\bar{a}, \bar{b}, \bar{h})$ where $\bar{a} \in\left\langle h, a, a^{2}\right\rangle$, $\bar{b} \in\left\langle t, t b_{\iota}, t b_{\imath}^{2}\right\rangle, \bar{h} \in\langle 1, h\rangle$. It is not difficult to see that the subgroup $T_{\iota}=\{\rho(a)$, $\left.\rho\left(b_{\iota}\right)\right\}$ permutes this set of 18 triplets amongst themselves in precisely the same way that $S_{1}=\{\rho(x), \rho(y)\}$ permutes the corresponding triplets ${ }^{1}$ in $P_{1}$. Since $S_{1}$ is soluble of length 2 the elements of the second derived group $T_{\iota}^{\prime \prime}$ of $T_{\iota}$ all map the 18 triplets under consideration identically. This argument clearly holds for any element $t(\neq 1)$ of $C_{\iota}$.

We now consider the effect of $\rho(a), \rho\left(b_{\iota}\right)$ on the 18 triplets $\left(\bar{a}, b^{*}, \bar{h}\right)$ where $\bar{a}, \bar{h}$ are as above and $b^{*} \in\left\langle h, b_{\imath}, b_{\imath}^{2}\right\rangle$. Clearly $T_{\imath}$ permutes this set of triplets amongst themselves in precisely the same way that $S_{2}$ permutes the corresponding set of triplets in $P_{2}$. Since $S_{2} \cong A_{9}, S_{2}=S_{2}^{\prime \prime}$. Thus any element of $S_{2}$ can be written as a product of commutators of the form $[[\alpha, \beta],[\gamma, \delta]]$ where each of $\alpha, \beta, \gamma, \delta$ is a product of $\rho(x), \rho(y)$ and their inverses.

If we now consider the corresponding elements in $T_{\iota}^{\prime \prime}$ (obtained by replacing

1 That is the set of 18 triplets $(\bar{x}, \bar{y}, \bar{k})$ where $\bar{x} \in X_{1}, \bar{y} \in Y_{1}$ and $\bar{k} \in\langle 1, k\rangle$. 
$\rho(x)$ by $\rho(a)$ and $\rho(y)$ by $\left.\rho\left(b_{\iota}\right)\right)$ the above considerations show that $T_{\iota}^{\prime \prime}$ generates a copy of $A_{9}$ on the $\left(\bar{a}, b^{*}, \bar{h}\right)$ and maps the $(\bar{a}, \bar{b}, \bar{h})$ identically.

Finally we select a subset of the set of all triplets on which $Q_{2}$ acts and label them as follows.
(1) $\cdots(h, h, 1)$
$\left(1^{\prime}\right) \cdots(h, h, h)$
(2) $\cdots\left(a^{2}, h, h\right)$
$\left(2^{\prime}\right) \cdots(a, h, 1)$
(3) $\cdots(a, h, h)$
$\left(3^{\prime}\right) \cdots\left(a^{2}, h, 1\right)$

and, for each integer $n=1,2,3, \cdots$ label

$$
\begin{array}{ll}
(6 n-2) \cdots\left(h, b_{n}^{2}, h\right) & \left(6 n-2^{\prime}\right) \cdots\left(h, b_{n}, 1\right) \\
(6 n-1) \cdots\left(a^{2}, b_{n}^{2}, 1\right) & \left(6 n-1^{\prime}\right) \cdots\left(a, b_{n}, h\right) \\
(6 n) \cdots\left(a, b_{n}^{2}, 1\right) & \left(6 n^{\prime}\right) \cdots\left(a^{2}, b_{n}, h\right) \\
(6 n+1) \cdots\left(h, b_{n}, h\right) & \left(6 n+1^{\prime}\right) \cdots\left(h, b_{n}^{2}, 1\right) \\
(6 n+2) \cdots\left(a^{2}, b_{n}, 1\right) & \left(6 n+2^{\prime}\right) \cdots\left(a, b_{n}^{2}, h\right) \\
(6 n+3) \cdots\left(a, b_{n}, 1\right) & \left(6 n+3^{\prime}\right) \cdots\left(a^{2}, b_{n}^{2}, h\right) .
\end{array}
$$

Now given any integer $m>3$ write $m=6 n+r$ where $-2 \leqq r \leqq 3$. The above considerations showed that $T_{n}^{\prime \prime}=\left\{\rho(a), \rho\left(b_{n}\right)\right\}$ generates a copy of $A_{9}$ on (1), (2), (3), $(6 n-2), \cdots(6 n+3) ;\left(1^{\prime}\right),\left(2^{\prime}\right),\left(3^{\prime}\right),\left(6 n-2^{\prime}\right) \cdots,\left(6 n+3^{\prime}\right)$ and leaves all other triplets invariant. In fact it is not difficult to check that $T_{n}^{\prime \prime}$ (and hence $\left.T_{n}\right)$ contains each of the permutations of the form $(12 k)\left(1^{\prime} 2^{\prime} k^{\prime}\right)$ where $k \in\langle 6 n-2, \cdots, 6 n+3\rangle$ (that is, a permutation moving $1,2, k, 1^{\prime}, 2^{\prime}, k^{\prime}$ as shown and fixing all the other triplets on which $Q_{2}$ acts), and also the permutation (1 123 ) $\left(1^{\prime} 2^{\prime} 3^{\prime}\right)$. Hence in $Q_{2}$ the subgroup $W_{2}=\left\{\rho(a), \rho\left(b_{1}\right), \rho\left(b_{2}\right), \cdots\right\}$ contains all permutations of the form $(12 m)\left(1^{\prime} 2^{\prime} m^{\prime}\right)$ with $m \geqq 3$. Hence $W$ contains a subgroup isomorphic to the alternating group on a countable infinity of symbols thus proving that $Q_{2}$ is not residually finite.

\section{References}

[1] R. J. Gregorac, 'Residual finiteness of permutational products', J. Aust. Math. Soc. 10 (1969), 423-428.

[2] K. W. Gruenberg, 'Residual properties of infinite soluble groups', Proc. London Math. Soc. 7 (1957), $29-62$.

[3] B. H. Neumann, 'Permutational products of groups', J. Aust. Math. Soc. 1 (1960), 299-310.

School of Mathematics

The University

Leeds, England.

LS2 9JT. 